\title{
Carbon nanotubes suspended in ethylene glycol yield nanofluids with enhanced heat transfer properties
}

\author{
H. B. Cheng ${ }^{1}$, W. T. $\mathrm{Ma}^{1}$, W. Hu${ }^{2}$, J. F. Wu ${ }^{1}$, J. Xiao ${ }^{1}$, \\ Z. B. Chen ${ }^{1}$, Q. J. Zhang ${ }^{1} \&$ N. M. Wereley ${ }^{2}$ \\ ${ }^{1}$ State Key Laboratory of Advanced technology for Materials Synthesis \\ and Processing, Wuhan University of Technology, China \\ ${ }^{2}$ Department of Aerospace Engineering, University of Maryland, USA
}

\begin{abstract}
Nanofluids (NFs) consisting of carbon nanotubes (CNTs) suspended in ethylene glycol (EG) were synthesized, and their stability and thermal conductivity enhancement rate were investigated. Stability of the CNT/EG-based NFs was enhanced by adding gum Arabic (GA). The increase in suspension stability was experimentally characterized using static and centrifugal testing processes. The mechanisms of GA in enhancing the fluid stability were characterized and the optimal mass ratio of GA to CNT was found to be about $11 \%$ by weight. Thermal conductivity was measured for varying CNT volume fraction under different fluid temperatures, and a nearly linear relationship between the thermal conductivity and the CNT volume fraction and temperature was observed.
\end{abstract}

Keywords: carbon nanotubes, nanofluids, gum Arabic, stability, thermal conductivity enhancement.

\section{Introduction}

Nanofluids (NFs) are a new type of enhanced heat transfer fluid that contains solid nanoparticles in a base fluid. Compared with conventional heat transfer fluids, such as silicone oil and ethylene glycol (EG), NFs are expected to have higher heat transfer efficiency [1]. Carbon nanotube-based (CNT-based) nanofluids are a most promising enhanced heat transfer fluid because CNTs have a higher thermal conductivity and relatively lower mass density than other 
nanoparticles such as copper $(\mathrm{Cu})$, aluminium oxide $\left(\mathrm{Al}_{2} \mathrm{O}_{3}\right)$ and titanium dioxide $\left(\mathrm{TiO}_{2}\right)[2,3]$. In fact, experimental results have shown that CNT-based nanofluids possess significantly higher enhanced thermal conductivity [3-5]. For example, Choi et al. [2] reported an enhancement of $160 \%$ for a CNT concentration of 1 vol $\%$ dispersed in poly-( $\alpha$ olefin) oil. However, pristine CNTs, which are chemically inert and have a large aspect ratio, have a strong tendency to entangle and to rapidly aggregate due to their large hydrophobic surface area [6]. As such, the stability of the CNT-based NFs is poor because the CNTs quickly stratify and deposit as sediment in the bottom of the container. Such rapid sedimentation, which can clog any flow channels, seriously decays heat transfer effectiveness and renders practical engineering applications infeasible [7, 8]. Therefore, improving the sedimentation stability of CNT-based NFs is the key challenge to enable successful engineering applications of these CNT-based NFs [9]. To develop an efficient method to mitigate these disadvantages, we investigated the effects of gum Arabic (GA) on the sedimentation stability and the thermal conductivity enhancement rate of the CNT/EG-based NFs. The sedimentation stability of the fluid was evaluated via observation of sedimentation in a static column, as well as a centrifugation process, and the thermal conductivity of the fluid was measured using a KD2Pro Thermal Properties Analyzer. Moreover, mechanisms enhancing NF stability were studied.

\section{Preparation of fluids}

The pristine (multiwalled) carbon nanotubes (p-CNTs), purchased from Nanjing Ji Cang Nano Technology Inc., were produced by a catalytic vapor deposition process. The parameters of $\mathrm{p}$-CNTs provided by the manufacturer are listed in Table 1. As can be seen in Table 1, the average outside diameter and the average length of p-CNTs are respectively $11 \mathrm{~nm}$ and $10 \mu \mathrm{m}$. Also, p-CNTs have a high aspect ratio exceeding 900. Furthermore, a small number of impurities exist in p-CNTs. The GA-absorbed CNT samples were obtained with a CNT/EG-based $\mathrm{NF}$, through vacuum suction filtration with a $0.22 \mu \mathrm{m}$ PTFE membrane, washed with DI water until the filtrate became nearly neutral and dried in a vacuumed environment at $333 \mathrm{~K}$.

Table 1: The parameters of $\mathrm{p}-\mathrm{CNT}$ s provided by the manufacturer.

\begin{tabular}{|c|c|c|}
\hline Parameters & Units & Values \\
\hline Average out diameter (OD) & $\mathrm{nm}$ & 11 \\
\hline Average length & $\mu \mathrm{m}$ & 10 \\
\hline Purity & $\%$ & $>95$ \\
\hline Metal oxides(impurity) & $\%$ & $<5$ \\
\hline Tap density & $\mathrm{g} / \mathrm{cm}^{3}$ & $0.013-0.15$ \\
\hline
\end{tabular}


CNT/EG-based nanofluids were prepared using a two-step method. A weighted sample of p-CNT powders and GA was dispersed into ethylene glycol (EG) using magnetic force agitation. The suspensions were further homogenized in an ultrasonic bath $(150 \mathrm{~W}, 40 \mathrm{~Hz})$ for a period of 60 minutes, and the temperature of the bath was maintained under $40^{\circ} \mathrm{C}$ with the help of a circulating water bath.

\section{NF stability and thermal conductivity}

\subsection{Dispersion stability}

The morphology of p-CNTs was characterized by a scanning electron microscope (SEM, Hitachi S-4800), as shown in Figure 1. It can be seen that impurities, such as catalyst particles, entanglements and aggregates, exist in pCNTs, and they are the key factors that make it difficult for p-CNTs to be dispersed into the base liquids.

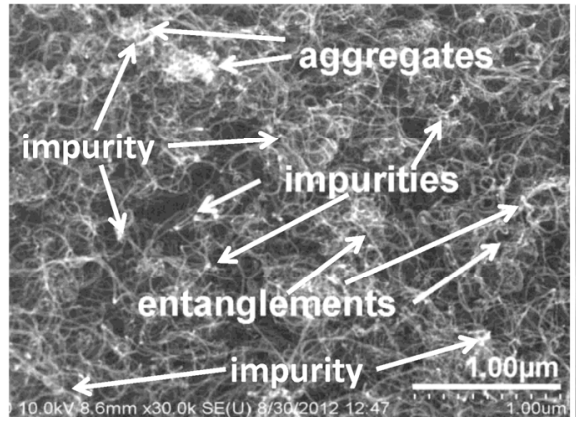

(a)

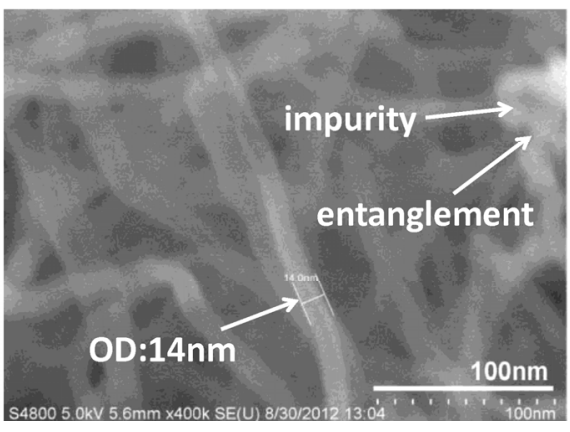

(b)

Figure 1: $\quad$ SEM micrographs of the pristine multiwalled carbon nanotubes with $\times 30 \mathrm{k}$ magnification (a) and $\times 400 \mathrm{k}$ magnification (b), respectively.

Figure 2 shows the sedimentations of the CNT/EG-based NFs made with $0.5 \mathrm{vol} \% \mathrm{p}$-CNTs without GA in a standing static column. It can be clearly seen that $\mathrm{p}-\mathrm{CNTs}$ are difficult to disperse in the base liquid since the p-CNTs in the nanofluids formed agglomerates (observable to the naked eye) after standing for only 5 minutes and were rapidly deposited at the bottom of the vial after standing for 70 minutes with very few p-CNTs remaining in the fluid column. This is in accordance with the findings in previous reports $[6,7]$. 


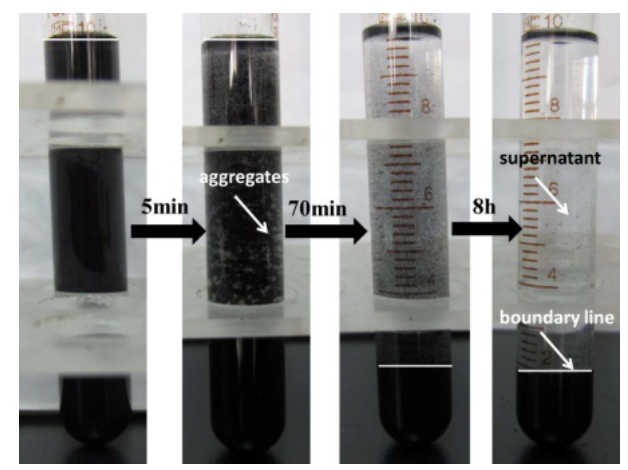

Figure 2: Sedimentation of the CNT/EG-based NF made with 0.5 vol\% CNTs after standing 0, 5, 70 minutes and 8 hours, respectively.

In order to study the effect of GA on the stability of CNT/EG-based NFs, a different amount of GA was added into the CNT/EG-based NF, made with 0.5 vol $\%$ CNTs according to the mass ratio of GA to CNTs ranging from $8 \%$ to $15 \%$. The comparison study of dispersion stability was conducted after the fluids were prepared and stood still for 3 weeks. Figure 3 depicts the sedimentation of the $0.5 \mathrm{vol} \% \mathrm{CNT} / \mathrm{EG}$-based NFs with GA concentrations of 8, 9, 10, 11, 12 and 15 $\mathrm{wt} \%$, respectively after standing for 3 weeks. The dispersion stability of the $\mathrm{CNT} / \mathrm{EG}$-based NFs increases first with the concentration of GA but decreases as the percentage of the GA increases further. The most stable CNT/EG-based NFs is the fluid made with $11 \mathrm{wt} \% \mathrm{GA}$, in which excellent homogeneity was obtained and no separated fluid layer and no sedimentation was observed. To further investigate the feasibility of the $0.5 \mathrm{vol} \%$ CNT/EG-based NFs with 11 wt $\%$ GA applied in engineering applications, high speed centrifugation was adopted to evaluate the fluid's dispersion stability under extreme conditions. High speed centrifugation was conducted for 5 hours at $10,000 \mathrm{rpm}$ to the prepared CNT/EG-based NFs in a plastic centrifugation tube. It showed that the CNT/EG NFs maintained consistent homogeneity with neither supernatant fluid appearing, nor noticeable sedimentation. Thus, we concluded that the addition of GA improves the dispersion stability of the CNT/EG-based NFs and the amount of GA needs to be optimized to obtain the most stable CNT/EG-based NFs. The optimal mass ratio of GA to CNTs was $11 \mathrm{wt} \%$.

The mechanism of GA in improving the stability of CNTs/EG NFs is also analyzed. GA is a surfactant that can be absorbed on the surface of CNTs to enable steric separation to hinder the formation of CNT agglomerates; this led to increased dispersion stability of the CNT/EG-based NFs. Ideally, monolayer adsorption would be the best to obtain optimal stability. However, there exists a critical GA concentration since the surface area is limited for a given CNT concentration. As the amount of GA exceeds the critical value, the excess amount of GA can form multilayer absorption, which increases the propensity for the carbon nanotubes to agglomerate. Experiments were conducted to further investigate the effect of GA concentration. Two GA-absorbed CNT samples 
were prepared using two fluid samples, i.e. $11 \mathrm{wt} \%$ GA CNT/EG-based NFs and $15 \mathrm{wt} \% \mathrm{CNT} / \mathrm{EG}$-based NFs, and the microstructure and morphology of the prepared GA-absorbed CNTs samples were observed using the SEM. As shown in Figure 4(a), most CNTs were nearly homogeneously dispersed in the sample made from the $11 \mathrm{wt} \%$ GA CNT/EG-based NFs and there exists few excess amounts of GA on the surface of the CNTs leading to few clumped CNTs. In contrast, in the sample made from the $15 \mathrm{wt} \%$ GA CNT/EG-based NFs (shown in Figure 4(b)), the excess amounts of GA interact with each other resulting in more clumped CNTs. The experimental observations as well as the analytical interpretation can be used to explain why the sedimentation stability of the CNT/EG-based NFs firstly increased and then decreased as GA concentration increased.

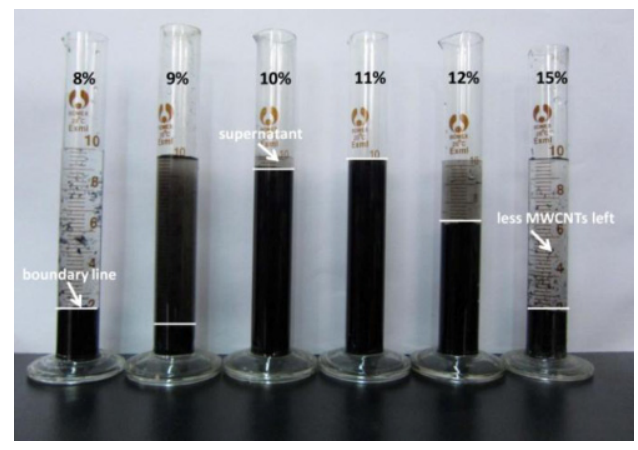

Figure 3: Sedimentation of the 0.5 vol\% CNT/EG-based NFs after standing for 3 weeks. GA concentration varied from adding GA in concentrations of $8,9,10,11,12$ and $15 \mathrm{wt} \%$.

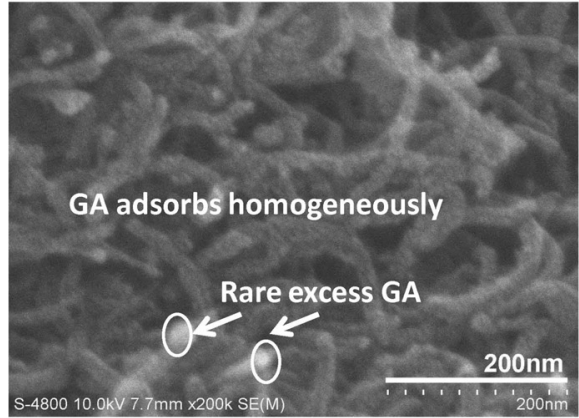

(a)

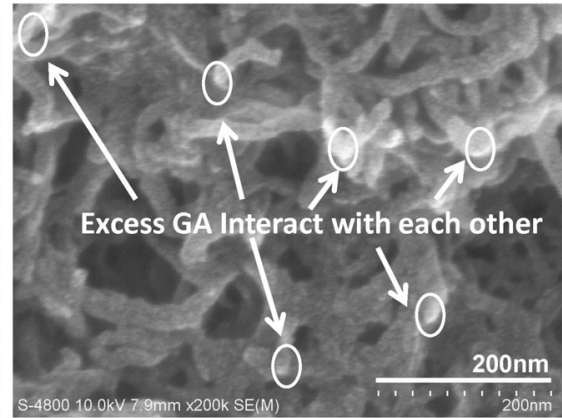

(b)

Figure 4: $\quad$ SEM micrographs of GA-absorbed CNTs samples made from the $11 \mathrm{wt} \%$ GA CNT/EG-based NFs (a) and made from the 15 wt $\%$ GA CNT/EG-based NFs (b). 


\subsection{Enhanced thermal conductivity}

\subsubsection{Effect of volume fraction of CNTs on the thermal conductivity}

In order to determine an optimal composition of CNT/EG-based NFs, the optimal mass ratio of GA to CNTs of $11 \mathrm{wt} \%$ was fixed as a standard and the volume fraction of CNTs was varied from 0.1 vol. $\%$ to 0.9 vol. $\%$ when preparing fluids. Nine CNT/EG-based NFs samples were prepared and their thermal conductivity was measured using a KD2 Pro analyzer (Decagon Devices Inc., USA). The analyzer operates using the principle of a transient line heat source method and complies with ASTM D5334-08 and IEEE442-1981 standards for determination of thermal conductivity. The KD2 Pro analyzer consists of a controller and a sensor. In order to obtain more accurate results, the time interval of each measurement was over 15 minutes so the testing sample can equilibrate from the previous measurement. The measurement was repeated at least five times for each sample and the average of the measurements was adopted. The measured thermal conductivity of the CNTs/EG NFs can be displayed on the screen of the controller, and can be transferred into a computer for data processing. In order to eliminate systematic error and evaluate the effect of volume fraction on thermal conductivity, the relationship diagrams between thermal conductivity enhancement rate $\left(k_{f}-k_{l}\right) / k_{l}$ and volume fraction were obtained, in which $k_{f}$ and $k_{l}$ refer to the thermal conductivity of CNTs/EG NFs and base liquid (EG) respectively. As shown in Figure 5, the enhancement rate $\left(k_{f}-k_{l}\right) / k_{l}$ increases approximately linearly with the volume fraction of CNTs, and is up to $14.18 \%$ at $20^{\circ} \mathrm{C}$ when the volume fraction of the CNTs is $0.9 \mathrm{vol} \%$.

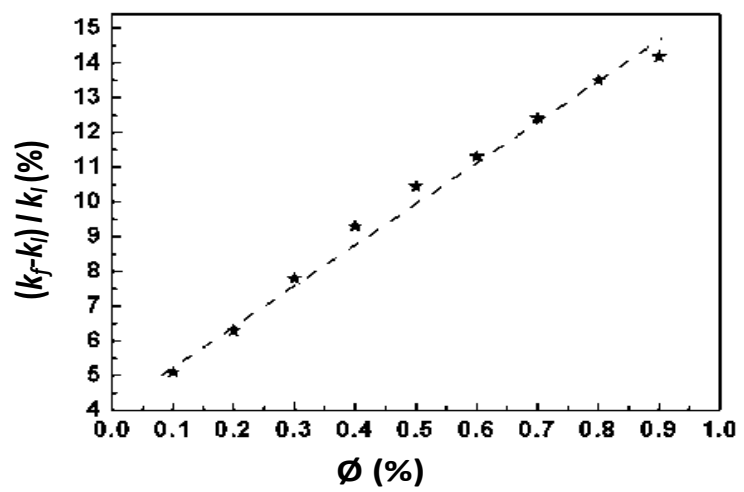

Figure 5: Thermal conductivity enhancement rate of CNT/EG-based NFs as a function of the volume fraction of CNTs at $20^{\circ} \mathrm{C}$.

\subsubsection{Effect of temperature on the thermal conductivity}

To explore the effect of temperature on thermal conductivity, a CNT/EG-based NF (volume fraction of CNTs 0.5 vol. $\%$, mass ratio of GA to CNTs equaling $11 \%$ ) was selected as a sample and a set of experiments performed as shown in Figure 6 was developed for measuring the thermal conductivity of CNTs/EG NFs under different temperatures. The relationship between the thermal 
conductivity enhancement rate $\left(k_{f}-k_{l}\right) / k_{l}$ and temperature was obtained as shown in Figure 7. It is shown that the thermal conductivity enhancement rate increases almost linearly with the temperature, i.e. from $10.64 \%$ to $20.21 \%$ as the temperature increases from $20^{\circ} \mathrm{C}$ to $60^{\circ} \mathrm{C}$. Interestingly, the thermal conductivity of the CNTs/EG NFs increases instead of declines as temperature increases, which differs from conventional heat transfer fluids and is especially useful for practical engineering applications.

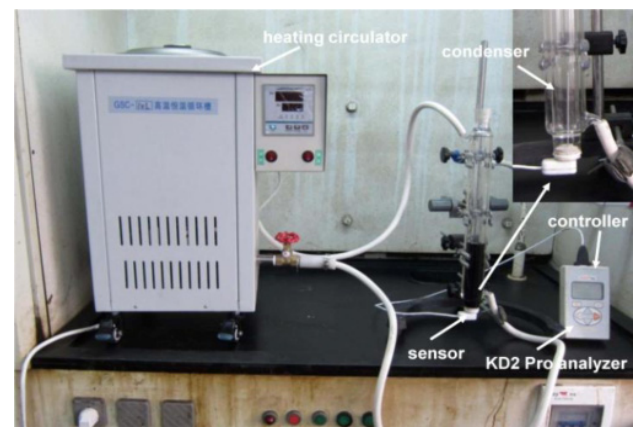

Figure 6: Experimental setup for measuring the thermal conductivity of CNT/EG-based NFs under different temperatures.

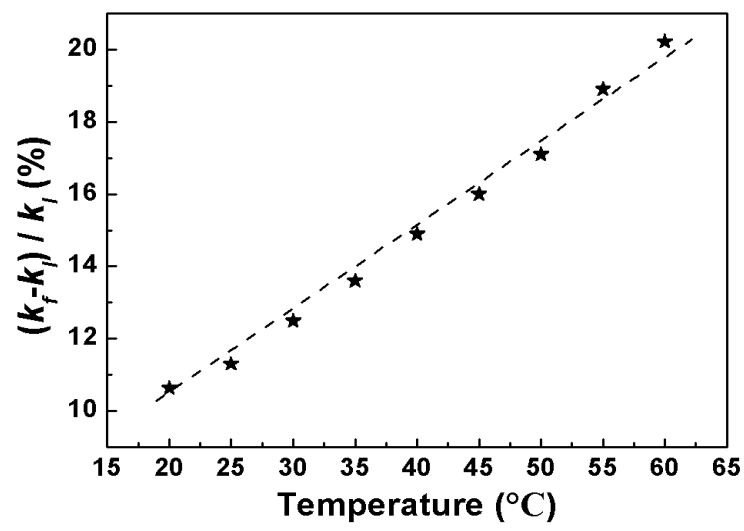

Figure 7: Relationship between thermal conductivity enhancement rate (kf$\mathrm{kl} / \mathrm{kl}$ and temperature for the CNT/EG-based NFs (volume fraction of CNTs equals $0.5 \mathrm{vol} \%$, mass ratio of GA to CNTs equals $11 \mathrm{wt} \%$ ).

\section{Conclusions}

Nanofluids (NFs) consisting of carbon nanotubes (CNTs) suspended in ethylene glycol (EG) were synthesized using gum Arabic as an additive. The stability and thermal conductivity enhancement rate of the fluid were measured. Experiments demonstrated that the gum Arabic increased the suspension stability of the fluid 
and the optimal mass ratio of GA to CNTs was about $11 \mathrm{wt} \%$. The CNT/EGbased NFs with the optimal GA component still exhibited a near homogeneous state after standing still for 3 weeks, as well as after high speed centrifugation at $10,000 \mathrm{rpm}$ for 5 hours. The thermal conductivity enhancement rate of the fluid increased approximately linearly with the volume fraction of CNTs and temperature. The enhancement rate is up to $14.18 \%$ for $0.9 \mathrm{vol} \% \mathrm{CNT} / \mathrm{EG}$-based $\mathrm{NFs}$ at $20^{\circ} \mathrm{C}$, and the enhancement rate for 0.5 vol. $\%$ CNTs/EG NF varied from $10.64 \%$ to $20.21 \%$ as the temperature increased from $20^{\circ} \mathrm{C}$ to $60^{\circ} \mathrm{C}$.

\section{Acknowledgement}

This work was financially supported by state key development program of basic research of China (2010CB227105) and Natural Science Foundation of China (NSFC 51372189).

\section{References}

[1] Kleinstreuer, C., Feng Y., Experimental and theoretical studies of nanofluids thermal conductivity enhancement: a review. Nanoscale Research Letters, 6(1), pp. 229-241, 2011.

[2] Choi, S.U.S, Zhang, Z.G., Yu, W., Lockwood, F.E., Grulke, E.A., Anomalous thermal conductivity enhancement in nanotube suspensions. Applied Physics Letters, 79 (14), pp. 2252-2254, 2001.

[3] Hwang, Y.J., Ahn, Y.C., Shin, H.S., Lee, C.G., Kim, G.T., Park, H.S., Lee, J.K., Investigation on characteristics of thermal conductivity enhancement of nanofluids. Current Applied Physics, 6(6), pp. 1068-1071. 2006.

[4] Harish, S., Ishikawa, K., Einarsson, E., Aikawa, S., Chiashi, S., Shiomi, J., Maruyama, S., Enhanced thermal conductivity of ethylene glycol with single-walled carbon nanotube inclusions. International Journal of heat and mass transfer, 55(13-14), pp. 3885-3890, 2012.

[5] Kumaresan, V., Velraj, R., Das, S. K., The effect of carbon nanotubes in enhancing the thermal transport properties of PCM during solidification. Heat and Mass Transfer, 48(8), pp. 1345-1355, 2012.

[6] Bandyopadhyaya, R., Nativ-Roth, E., Regev, O., Yerushalmi-Rozen, R., Stabilization of individual carbon nanotubes in aqueous solutions. Nano Letters, 2(1), pp. 25-28, 2002.

[7] $\mathrm{Yu}, \mathrm{W} ., \mathrm{Xie}, \mathrm{H}$. Q., A review on nanofluids: preparation, stability mechanisms and applications. Journal of Nanomaterials, 2012(1), pp. 1-17, 2012.

[8] Ghadimi, A., Saidur, R. and Metselaar, H. S. C., A review of nanofluid stability properties and characterization in stationary conditions. International Journal of Heat and Mass Transfer, 54(17-18), pp.4051-4068, 2011.

[9] Chen, J., Hamon, M.A., Hu, H., Chen, Y.S., Rao, A.M., Eklund, P.C., Haddon, R.C., Solution properties of single-walled carbon nanotubes. Science, 282(5386), pp. 95-98, 1998. 